\title{
Developing pathways for oral care in elders: evidence-based interventions for dental caries prevention in dentate elders
}

\author{
Elisa M. Ghezzi \\ University of Michigan School of Dentistry, Ann Arbor, MI, USA
}

doi: $10.1111 / \mathrm{ger} .12081$

Developing pathways for oral care in elders: evidence-based interventions for dental caries prevention in dentate elders

Background: Dental caries is becoming an ever-growing challenge as the number of elders maintaining their teeth increases. There is a need for low-cost, effective preventive interventions to retain natural teeth for elders.

Objective: The purpose of this article is to evaluate evidence based interventions for dentate elders, specifically the adjunct therapies of fluoride, chlorhexidine, xylitol, casein phosphopeptide-amorphous calcium phosphate, ozone, and herbal liquorice.

Results: Fluoride interventions have demonstrated prevention and remineralization of dental caries in elders. Systematic reviews of the literature are unable to establish definitive conclusions regarding the effectiveness of other adjunct therapies in dental caries prevention.

Conclusions: Further research with elders requires improved study design with well designed multicenter trials. Considerations for new strategies for research of the effectiveness of therapies to reduce dental caries include the development and evaluation of combinations of therapeutic interventions and dental caries management by risk assessment.

Keywords: geriatric dentistry, dental caries, ageing, prevention.

Accepted 13 September 2013

\section{Introduction}

Increasingly, elders are maintaining their teeth throughout their lifetime. Good daily oral hygiene, regular professional dental care, the ability to afford oral health care and exposure to fluoride are significant contributing factors to this trend. It is imperative that this investment be protected if elders expect to preserve their dentition. However, there are numerous challenges that elders face due to medical, physical and cognitive impairments. Oral hygiene practice is reduced following a decline in manual dexterity and inability to recall tasks of daily living. Salivary flow is reduced with an increase in medication intake secondary to medical conditions. A lifetime of periodontal insult results in bone loss and root exposure which more quickly succumbs to decay compared with enamel. Excessive sugar consumption, particularly in protein supplements, dry-mouth lozenges and sugar-rich food, further increases the compromised elder's risk to tooth decay ${ }^{1}$. Elders often have reduced funds and may lack dental insurance to cover the cost of routine preventive as well as restorative oral health care $^{2}$. Access to oral health services can also decline due to lack of transportation and the loss of independence secondary to physical and cognitive impairments.

The most common oral health problems experienced by elders are tooth loss, dental caries and periodontal disease. Between 1988-1994 and 1999-2004, the oral health status for U.S. seniors improved, as edentulism and periodontal disease declined $^{3}$. Mean periodontal recession, pocket depth and attachment loss declined across all major demographic subgroups of seniors indicating improved periodontal health in seniors. The prevalence of root caries in seniors decreased; however, the prevalence of coronal caries did not change. NHANES 1999-2004 data demonstrate 
that among adults younger than 64 years of age, the prevalence of decayed and filled tooth surfaces (DFS) was 19 surfaces, while in persons over the age of 65, the DFS prevalence was nearly 30 surfaces $^{3}$. A systematic review of studies of coronal caries in older adults aged $60+$ years revealed a caries rate of one new carious surface per person per year, which is equal to or higher than the rate in children ${ }^{4}$. Also, when compared to schoolchildren, the number of untreated decayed surfaces found in elders is greater ${ }^{4}$. Dental caries is an important oral health care issue that is not adequately prevented in elders and is becoming an ever-growing challenge as the number of elders maintaining their teeth increases.

A contributing factor to the challenge of reducing dental caries and the large DFS in elders is the surgical approach of dental practitioners in treating dental caries rather than prevention or pharmacotherapeutics. Historically, oral health care prevention has consisted of oral hygiene instruction (tooth brushing, flossing) and dietary modification. There is a need for low-cost, effective preventive interventions to retain natural teeth of elders especially when traditional methods are no longer adequate. Adjunct therapies have been utilised and evaluated in many studies with children. However, data on the impact of these therapies in elders are limited. The Centers for Disease Control and Prevention's 2001 Recommendations for Using Fluoride to Prevent and Control Dental Caries in the United States stated, 'Few studies evaluating the effectiveness of fluoride toothpaste, gel, rinse, and varnish among adult populations are available', and recommended research focus on the impact of different fluoride regimens on dental caries, specifically in adults aged $50+^{5}$.

The purpose of this article is to evaluate evidence-based interventions for dentate elders, specifically the adjunct therapies of fluoride, chlorhexidine, xylitol, casein phosphopeptideamorphous calcium phosphate, ozone and herbal liquorice.

\section{Fluoride}

The dental caries prevention mechanisms of fluoride include inhibition of bacterial metabolism of fermentable carbohydrates, inhibition of demineralisation of tooth structure and remineralisation of fluoride into tooth structure ${ }^{6}$. Studies on fluoride vary significantly by design. These sources of variation include concentration of fluorides, modes of delivery of fluoride (including water fluoridation, toothpaste, rinses, gels, foams and varnish) and types of administration (self- vs. professional application).

A systematic review examining the effectiveness of fluoride in preventing dental caries in adults found fluoride (self- and professionally applied or water fluoridation) annually averted 0.29 carious coronal and 0.22 carious root surfaces ${ }^{7}$ and concluded fluoride is effective among all adults supporting the development and implementation of fluoride programmes. However, the type of fluoride used in a programme influences the impact on dental caries reduction in elders ${ }^{8}$. Higher concentrations of fluoride toothpaste $(5000 \mathrm{ppm}$ of fluoride) and fluoride varnish (22 $600 \mathrm{ppm}$ of fluoride) have been shown to reverse and remineralise root caries significantly better than conventional toothpaste (1100 ppm and $14500 \mathrm{ppm}$ of fluoride) in adults, frail home-bound elders and elderly disabled nursing home residents ${ }^{9-11}$.

A systematic review of seventeen studies evaluated the effectiveness of professional and/or self-applied fluoride in the prevention or remineralisation of dental caries in moderate- and high-risk adults. All the studies demonstrated that the use of supplemental and professionally applied fluoride (NaF mouthrinses/1.1\% NaF paste or gel/Fluoride varnish) in moderate and high dental caries risk adults is effective in preventing and/or remineralising dental caries ${ }^{12}$. A daily $0.2 \%$ NaF mouthrinse has been shown to reduce the incidence of dental caries among institutionalised elders ${ }^{13}$.

Fluoride in combination with other modalities and therapies has been studied. Glass-ionomer restoratives containing fluoride have been suggested to inhibit secondary dental caries; however, a review of the evidence did not find support for this claim ${ }^{14}$. Daily intake of milk supplemented with 5 ppm fluoride may reverse primary root caries lesions ${ }^{15}$.

The focus of dental caries management by risk assessment is the framework for the development of dental caries management programmes ${ }^{16}$. Therapeutic management of dental caries is accomplished by reducing risk through the use of anticaries therapies that reduce pathological factors (antimicrobial therapies) and enhance protective factors (fluoride application). Interventions that have demonstrated similar effectiveness in reduction of root caries in elders include ${ }^{1}$ twicedaily use of $5000 \mathrm{ppm}$ fluoride toothpaste ${ }^{2}$, monthly application of 22600 ppm fluoride varnish $^{3}$, quarterly application of $1 \%$ chlorhexidine varnish ${ }^{4}$, quarterly application of $5 \% \mathrm{NaF}$ varnish and $^{5}$ annual application of $38 \%$ silver diamine fluoride (SDF) solution ${ }^{10,17,18}$. 


\section{Chlorhexidine}

Chlorhexidine is an agent known for reducing levels of bacteria including mutans streptococci $^{19}$ as well for demonstrating antiplaque and antigingivitis properties ${ }^{20}$. Although reduction in the numbers of bacteria and improvement in periodontal conditions is well documented, the impact of chlorhexidine in the reduction in dental caries has not been established ${ }^{21}$.

A systematic review of 22 studies from 1995 to 2003 found the evidence for chlorhexidine arresting root caries in dry-mouth patients and frail elderly subjects inconclusive calling for more well-designed studies ${ }^{20}$. This review also suggested that antimicrobial therapy may act differently for carious lesions located in different tooth surfaces (enamel/dentin/root). The effectiveness of chlorhexidine has been evaluated as a $0.12 \%$ mouthrinse as well as a varnish in multiple concentrations. The Trial to Enhance Elderly Teeth Health (TEETH) evaluating the impact of regular $0.12 \%$ chlorhexidine rinsing failed to identify a significant effect on incident dental caries experience on either coronal or root structure ${ }^{22}$.

A systematic review evaluating the impact of chlorhexidine varnish $(1 \%, 10 \%, 40 \%)$ on root caries concluded that weak evidence exists to suggest chlorhexidine varnish may be beneficial in elders and persons with xerostomia where regular professional tooth cleaning and oral hygiene instructions are lacking ${ }^{23}$. When evaluating the effectiveness of weekly application of $10 \%$ chlorhexidine varnish to reduce incidence of dental caries in adults with reduced salivary flow secondary to medication intake, the impact varied by tooth surface. The reduction in root caries was $41 \%$, while the total dental caries reduction was only $25 \%$, suggesting that the impact of chlorhexidine may depend on tooth surface ${ }^{19}$. The Prevention of Adult Caries Study of high-risk adults found that 10\% chlorhexidine diacetate varnish did not prevent the development of new coronal caries ${ }^{24}$. The conclusions from the current findings suggest that future studies of chlorhexidine varnish focus on prevention of root caries in high-risk populations. In addition, the inability of systematic reviews of studies with chlorhexidine to establish definitive conclusions stresses the necessity of improved study design with well-designed multicentre trials.

\section{Xylitol}

Substitution of sucrose with other sugar alcohols such as xylitol has been proposed for dental caries prevention as mutans streptococci are unable to metabolise xylitol. A review of the effect of noncariogenic sweeteners on the prevention of dental caries suggests that the replacement of sucrose with sorbitol and xylitol may significantly reduce the incidence of dental caries ${ }^{25}$. Xylitol is often recognised as superior to other sugar alcohols in its anticariogenic properties. A review of studies on the effectiveness of xylitol concluded that the regular use of xylitol is more likely to reduce numbers of mutans streptococci in saliva and plaque than regular use of sorbitol. However, not all studies on the effect of xylitol confirm its mutansreducing effect ${ }^{26}$. A randomised control trial evaluating the impact of xylitol rinse and chewing gum found that levels of mutans streptococci did not significantly reduce ${ }^{27}$. Data from multiple studies support that the administration of xylitol or combined xylitol and sorbitol gums and lozenges several times a day prevents dental caries ${ }^{26}$. However, the claim that xylitol has a dental caries-therapeutic effect or remineralisation properties is not substantiated ${ }^{26}$.

Studies of the effectiveness of xylitol in elders are lacking. In response to a call from the National Institutes of Health Consensus Development Conference on Diagnosis and Management of Dental Caries Throughout Life $^{28}$ for trials that demonstrate effectiveness of dental caries prevention interventions in adults, the xylitol for adult caries trial (X-ACT) was designed to test the hypothesis that the use of xylitol lozenges will reduce dental caries incidence in caries-active adults. Daily use of xylitol lozenges did not result in a statistically or clinically significant reduction in dental caries in high-risk adults over a 33month period ${ }^{29}$. More longitudinal, multicentre, placebo-controlled, double-blind, randomised clinical trials are required to determine the effectiveness of xylitol and other dental caries preventive measures.

\section{Casein phosphopeptide-amorphous calcium phosphate}

Casein phosphopeptide-amorphous calcium phosphate (CPP-ACP) nanocomplexes have been suggested to have anticariogenic properties. The proposed anticariogenic mechanism of CPP is its ability to localise and stabilise ACP at the tooth surface producing a highly water-soluble calcium phosphate phase. Prevention of enamel demineralisation and promotion of enamel remineralisation occur through the state of supersaturation of calcium and phosphate ions created at the enamel 
surface $^{30}$. Private practice use and prescription of CPP-ACP paste is common place with U.S. dentists, while the data to support its impact on dental caries remineralisation are lacking.

In an in vitro study evaluating the inhibitory effect of CPP-ACP paste, 1100 and $5000 \mathrm{ppm}$ fluoride pastes, and a combination of fluoride and CPP-ACP paste, the CPP-ACP paste alone did not show any effect on the inhibition of lesion progression $^{31}$. The combination of CPP-ACP paste with $900 \mathrm{ppm}$ fluoride may reduce demineralisation in orthodontic patients ${ }^{32}$. Currently, there is not sufficient scientific evidence of the remineralisation effectiveness of CPP-ACP paste alone. Recommendations for further studies of CPP-ACP method of application (lozenges, chewing gum, paste) in the prevention of demineralisation should include CPP-ACP in combination with fluoride and include longer treatment applications, extended exposure to the oral environment and increased follow-up measurements.

\section{Ozone}

Direct application of ozone gas to a carious lesion on the tooth surface has been proposed to reverse, arrest or slow the progression of dental caries. Components of the HealOzone ${ }^{\circledR}$ (CurOzone USA Inc., Aurora, ON, Canada) treatment also include the use of a remineralising solution and oral hygiene products containing fluoride. A systematic review of the effectiveness of ozone for the treatment of occlusal and root caries concluded that there is not yet enough evidence from published randomised control trials from which to judge ozone ${ }^{33}$. It suggested that further studies are required with adequate design, outcome measures and methods for statistical analyses. Concern was raised that ozone may be more expensive than current treatment modalities ${ }^{33}$.

\section{Herbal liquorice}

Glycyrrhiza uralensis, commonly known as liquorice root, has been shown to have antibacterial properties $^{34}$. A sugar-free, orange-flavoured herbal lollipop containing the antibacterial compound Glycyrrhiza uralensis has been developed to reduce oral S. mutans levels. The studies involved in the lollipop development demonstrated in adults its safety and effectiveness in reducing bacterial count and percentage over a 10-day period of twice-daily administration ${ }^{35}$. Twice-daily use of herbal liquorice lollipop significantly reduced the number and relative percentage of $S$. mutans in high-risk children during a 3-week intervention ${ }^{36}$. The results of these studies indicate that further randomised controlled trials are needed to determine the efficacy of the antibacterial properties as well as the impact of bacterial reduction in dental caries prevention. As the liquorice extract did not interfere with the anticariogenic activity of fluoride or xylitol, it should be studied in conjunction with other therapies ${ }^{35}$.

\section{Salivary stimulants and replacement}

Xerostomia due to medication intake is common in elders as many have been prescribed multiple medications for medical diagnoses ${ }^{37}$. Reduced salivary flow can exacerbate an elder's high risk for dental caries. Salivary flow can be stimulated with sugarfree candies, gums and mints. Increased fluid intake, particularly water, should be encouraged. Artificial salivary replacements can be applied as gels or sprays ${ }^{38}$. Pilocarpine and cevimeline are secretagogues that can be prescribed to stimulate salivary production; however, the side effects of these medications often preclude their use. Substitution of xerostomic medication or alteration of medication administration can reduce the impact of the medication on the salivary flow ${ }^{39}$.

\section{Discussion}

This evaluation of evidence-based interventions for dentate elders considered the evidence for the effectiveness of adjunct therapeutic interventions in the reduction in dental caries. Most of the systematic reviews of these adjunct therapies concluded that, with the exception of fluoride, the current body of evidence is too weak to establish definitive claims of effectiveness. Twetman ${ }^{20}$ proposed a need for 'the scientific community to initiate clinical studies to confirm efficacy and safety of non-surgical treatment of dental caries. Not more, but better ones: well-designed, multicentre trials with antibacterial intervention according to a standard protocol in select representative study groups with enough power to ensure firm conclusions'. As clinical trials involving elders is limited, future investigations should focus on this study group.

Although fluoride is effective in dental caries reduction in elders, concerns for its safety preclude patient acceptance and use. The most effective fluoride concentrations require a prescription for administration. However, the requirement for prescription can inhibit the accessibility and affordability of the product. For a prescription 
medication to be affordable for a patient, it often needs to be included as a covered medication in an insurance plan. In a long-term care facility, a fluoride option often needs to be included in a pharmacy formulary for it to be accessible to the residents, and there can be an increased cost for the administration of medications. Due to its classification as a prescribed medication, family and non-staff caregivers are often prohibited from administering the product.

\section{Conclusion}

Research priorities for dental caries prevention must include dentate elders. New strategies for research of the effectiveness of therapies to reduce dental caries include the development and evaluation of combinations of therapeutic interventions $^{28}$ and dental caries management by risk assessment ${ }^{16,28}$. There is need to provide clinical evidence that scientifically based dental caries risk assessment with corresponding aggressive preventive measures (i.e. targeted antibacterial and fluoride therapy based on salivary microbial and fluoride levels) and conservative restorations would result in reduced dental caries increment ${ }^{16}$. To achieve compliance and efficacy, consumer products and homecare procedures need to be developed and become more accessible ${ }^{20}$. Studies in patients at risk for dental caries and vulnerable groups are needed to increase knowledge and self-care practices by communicating preventive health messages and increasing motivation $^{20}$.

\section{Conflicts of interest}

None declared.

\section{References}

1. Steele JG, Sheiham A, Marcenes W, Fay N, Walls AW. Clinical and behavioural risk indicators for root caries in older people. Gerodontology 2001; 18: 95-101.

2. White BA. Factors influencing demand for dental services: population, demographics, disease, insurance. J Dent Educat 2012; 76: 996-1007.

3. Dye BA, Tan S, Smith V, Lewis BG, Barker LK, Thornton-Evans G et al. Trends in oral health status: United States, 1988-1994 and 19992004. National Center for Health Statistics. Vital Health Stat 2007; 248: 1-92.

4. Griffin So, Griffin PM, Swannm JL, Zlobin N. New coronal caries in older adults: implications for prevention. J Dent Res 2005; 84: 71520.

5. Anonymous. Recommendations for using fluoride to prevent and control dental caries in the United States. Centers for Disease Control and Prevention. MMWR Recomm Rep 2001; 50 (RR-14): 1-42.

6. Featherstone JDB. The science and practice of caries prevention. $J \mathrm{Am}$ Dent Assoc 2000; 131: 887-99.

7. Griffin SO, Regnier E, Griffin PM, Huntley V. Effectiveness of fluoride in preventing caries in adults. J Dent Res 2007; 86: 410-5.

8. Fure S, Gahnberg L, Birkhed D. A comparison of four home-care fluoride programs on the caries inci- dence in the elderly. Gerodontology 1998; 15: 51-60.

9. Baysan A, Lynch E, Ellwood R, Davies R, Petersson L, Borsboom P. Reversal of primary root caries using dentifrices containing 5,000 and 1,100 ppm fluoride. Caries Res 2001; 35: 41-6.

10. Ekstrand K, Martignon S, HolmPedersen P. Development and evaluation of two root caries controlling programmes for home-based frail people older than 75 years. Gerodontology 2008; 25: 67-75.

11. Ekstrand KR, Poulsen JE, Hede B, Twetman S, Qvist V, Ellwood RP. A randomized clinical trial of the anti-caries efficacy of 5,000 compared to $1,450 \mathrm{ppm}$ fluoridated toothpaste on root caries lesions in elderly disabled nursing home residents. Caries Res 2013; 47: 391-8.

12. Gibson G, Jurasic MM, Wehler C, Jones JA. Supplemental fluoride use for moderate and high caries risk adults: a systematic review. J Pub Health Dent 2011; 71: 171-84.

13. Wyatt CCL, MacEntee MI. Caries management for institutionalized elders using fluoride and chlorhexidine mouthrinses. Community Dent Oral Epidemiol 2004; 32: 322-8.

14. Randall RC, Wilson NH. Glass-ionomer restoratives: a systematic review of a secondary caries treatment effect. J Dent Res 1999; 78: 628-37.

15. Petersson LG, Magnusson K, Hakestam U, Baigi A, Twetman
S. Reversal of primary root caries lesions after daily intake of milk supplemented with fluoride and probiotic lactobacilli in older adults. Acta Odontol Scand 2011; 69: 321-7.

16. Featherstone JD, White JM, Hoover CI, Rapozo-Hilo M, Weintraub JA, Wilson RS et al. A randomized clinical trial of anticaries therapies targeted according to risk assessment (caries management by risk assessment). Caries Res 2012; 46: $118-29$.

17. Tan HP, Lo EC, Dyson JE, Luo Y, Corbet EF. A randomized trial on root caries prevention in elders. J Dent Res 2010; 89: 1086-90.

18. Walls AWG, Meurman JH. Approaches to caries prevention and therapy in the elderly. Adv Dent Res 2012; 24: 36-40.

19. Banting DW, Papas A, Clark DC, Proskin HM, Schultz M, Perry R. The effectiveness of $10 \%$ chlorhexidine varnish treatment on dental caries incidence in adults with dry mouth. Gerodontology 2000; 17: 6776.

20. Twetman S. Antimicrobials in future caries control?: a review with special reference to chlorhexidine treatment. Caries Res 2004; 38: 223 9.

21. Persson RE, Truelove EL, LeResche L, Robinovitch MR. Therapeutic effects of daily or weekly chlorhexidine rinsing on oral health of a geriatric population. Oral Surg 
Oral Med Oral Pathol 1991; 72: 184 91.

22. Wyatt CCL, Maupome G, Hujoel PP, MacEntee MI, Persson GR, Persson RE et al. Chlorhexidine and preservation of sound tooth structure in older adults. Caries Res 2007; 41: 93-101.

23. Slot DE, Vaandrager NC, Van Loveren C, Van Palenstein Helderman WH, Van der Weijden GA. The effect of chlorhexidine varish on root caries: a systematic review. Caries Res 2011; 45: 162-73.

24. Papas AS, Vollmer WM, Gullion CM, Bader J, Laws R, Fellows J et al. Efficacy of chlorhexidine varnish for the prevention of adult caries: a randomized trial. J Dent Res 2012; 91: 150-5.

25. Hayes C. The effect of non-cariogenic sweeteners on the prevention of dental caries: a review of the evidence. J Dent Educat 2001; 65: 1106-9.

26. Van Loveren C. Sugar alcohols: what is the evidence for caries-preventive and caries-therapeutic effects? Caries Res 2004; 38: 286-93.

27. Hildebrandt G, Lee I, Hodges J. Oral mutans streptococci levels following use of a xylitol mouth rinse: a double-blind, randomized, controlled clinical trial. Spec Care Dentist 2010; 30: 53-8.

28. Anonymous. Diagnosis and management of dental caries throughout life. National Institutes of Health Consensus Development Conference statement, March 26-28, 2001. J Dent Educ 2001; 65: 1162-8.

29. Bader JD, Vollmer WM, Shugars DA, Gilbert GH, Amaechi BT, Brown JP et al. Results from the xylitol for adult caries trial (XACT). J Am Dent Assoc 2013; 144: 21-30.

30. Reynolds EC, Cai F, Shen P, Walker GD. Retention in plaque and remineralization of enamel lesions by various forms of calcium in a mouthrinse or sugar-free chewing gum. J Dent Res 2003; 82: 206-11.

31. Pulido MT, Wefel JS, Hernandez MM, Denehy GE, Guzman-Armstrong S, Chalmers JM et al. The inhibitory effect of MI paste, fluoride and a combination of both on the progression of artificial caries-like lesions in enamel. Operative Dent 2008; 33: 550-5.

32. Robertson MA, Kau CH, English JD, Lee RP, Powers J, Nguyen JT. MI Paste Plus to prevent demineralization in orthodontic patients: a prospective randomized controlled trial. Am J Orthod Dentofacial Orthop 2011; 140: 660-8.

33. Brazzelli $\mathbf{M}$, McKenzie L, Fielding S, Fraser C, Clarkson J, Kilonzo $\mathbf{M}$ et al. Systematic review of the effectiveness and cost-effectiveness of HealOzone for the treatment of occlusal pit/fissure caries and root caries. Health Technol Assess 2006; 10: iii-80.

34. He J, Chen L, Heber D, Shi W, Lu Q. Antibacterial compounds from
Glycyrrhiza uralensis. J Nat Prod 2006; 69: 121-4.

35. Hu C, He J, Eckert R, Wu X, Li L, Tian Y et al. Development and evaluation of a safe and effective sugarfree herbal lollipop that kills cavitycausing bacteria. Int J Oral Sci 2011; 3: 13-20.

36. Peters MC, Tallman JA, Braun TM, Jacobson JJ. Clinical reduction of S. mutans in pre-school children using a novel liquorice root extract lollipop: a pilot study. Eur Arch Paediatr Dent 2010; 11: 274-8.

37. Liu B, Dion MR, Jurasic MM, Gibson G, Jones JA. Xerostomia and salivary hypofunction in vulnerable elders: prevalence and etiology. Oral Surg Oral Med Oral Pathol Oral Radiol 2012; 114: 52-60.

38. Visvanathan V, Nix P. Managing the patient presenting with xerostomia: a review. Int J Clin Pract 2010; 64: 404-7.

39. Ship JA, Pillemer SR, Baum BJ. Xerostomia and the geriatric patient. J Am Geriatr Soc 2002; 50: 535-43.

\section{Correspondence to:}

Elisa M. Ghezzi, DDS, PhD, 26024 Pontiac Trail, South

Lyon, MI 48178, USA.

Tel.: +2484864828

Fax: +8557782780

E-mail: eghezzi@umich.edu 